\title{
Research on muffler performance control by arrangement of Helmholtz resonators
}

\author{
Yannan $\operatorname{Han}^{1, a}$, Haifeng Iv ${ }^{1, b}$ \\ ${ }^{1}$ School of Mechanical and Power Engineering, North University of China, Taiyuan, 030051, China. \\ a286267631@qq.com, b Ivhaifeng@nuc.edu.cn
}

Keywords: Helmholtz resonator; Muffler; Transmission loss; Finite element method

\begin{abstract}
The Helmholtz resonator is widely used as an effective way to reduce noise around the resonance frequency. But the resonator is a single degree of freedom and has a narrow resonance frequency band which determined by its geometry. This paper used three-dimensional finite element method to calculate the acoustic characteristics of different arrangement of the Helmholtz resonant. It is can be found that 2 Helmholtz resonators connected in parallel, the narrow-band not be changed and the performance of muffler is enhanced. As for the 2 degrees of freedom Helmholtz resonator has two resonance frequencies, and the narrow-band has been broaden. From the simulation, the performance of muffler has no significant change with distance change, but the effect of two Helmholtz resonators has been changed by the angle between the two Helmholtz resonators.
\end{abstract}

\section{Introduction}

Noise is the by-product of the industrial society, which is regarded as one of the three public hazards in the world today ${ }^{[1]}$. The Helmholtz resonator (HR) is widely used as an effective way to reduce noise around the resonance frequency ${ }^{[2]}$. While the disadvantage of HR is its effective frequency range is restricted by its resonance frequency, which is decided by the physical dimensions of neck area, neck length, and cavity volume. It is clear that the resonant frequency is fixed when the geometry of the resonator fixed. There are 2 method to optimize the effect of Helmholtz resonator, one is changing the geometry of the resonator or the arrangement of Helmholtz resonators, anther is designing a tuned Helmholtz resonator. This paper chooses the former, which using multiple Helmholtz resonator to optimize the performance by changing the arrangement of mufflers.

\section{Theoretical analysis}

Helmholtz resonator is widely used to attenuate the narrow-band low-frequency noise ${ }^{[3]}$. In view of the low frequencies of interest in this paper, the geometrical dimensions of HR considered here are significantly smaller than the fluid wave length. The Physical dimensions is shown in table1. Then the model of Helmholtz resonator can be established by lumped parameter theory. The mass significant for oscillation of single Helmholtz resonator in air medium is concentrated in the neck of the resonator and the volume of resonator acts as a spring, and the mechanical-acoustical analogy for single Helmholtz resonator in air medium which is a mass-spring system ${ }^{[3]}$. Assuming that flow can be neglected, and the wave field in the duct is isentropic.

The resonant frequency of the resonator can be expressed as

$$
f_{0}=\frac{c_{0}}{2 \pi} \sqrt{\frac{\pi r^{2}}{(t+\Delta t) v}}
$$

where $t$ and $r$ are the thickness and the radius of the neck, respectively, $\Delta t$ is the sum of the end correction at the inner and outer neck ends, $c_{0}=343 \mathrm{~m} / \mathrm{s}$ is sound velocity in medium, and $\mathrm{v}$ is the cavity volume. 


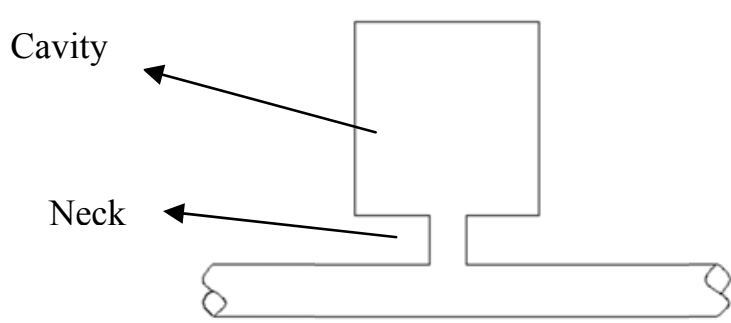

a) The structure of HR

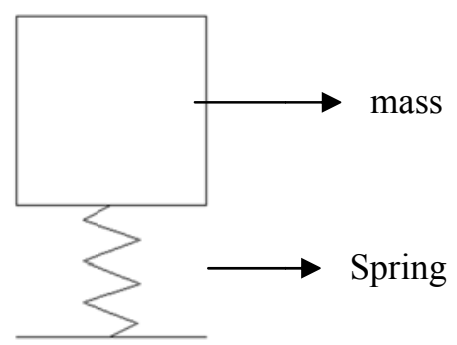

b) Equivalent mechanical system

Fig.1 Structure and Equivalent mechanical system of Helmholtz resonator

The transfer matrix method (also called the transmission matrix or the four-pole parameter representation) has been widely used in electrical and structural engineering ${ }^{[4]}$. With the transfer matrix method, complex circuits or structures can be simply regarded as "black boxes" with one input port and one output port. The interconnection of parallel, series-parallel, and parallel-series network combinations can then be handled by simple liner addition or multiplication of the transfer matrix.

The $P-Q$ transfer matrix is given by

$$
\left[\begin{array}{l}
P_{1} \\
Q_{1}
\end{array}\right]=\left[\begin{array}{ll}
T_{11} & T_{12} \\
T_{21} & T_{22}
\end{array}\right]\left[\begin{array}{l}
P_{2} \\
Q_{2}
\end{array}\right]
$$

Where $P$ is the acoustic pressure, $Q$ is the volume velocity and $T_{11}, T_{12}, T_{21}, T_{22}$ are the four pole parameters of the system.

If the temperature remains constant in the acoustic system, we can choose $\rho_{0} c_{0} U$ as the flow variable instead of the $Q$. where $\rho_{0} c_{0}$ is the characteristic acoustic impedance at this temperature. And the transfer matrix is

$$
\left[\begin{array}{c}
P_{1} \\
\rho_{0} c_{0} U_{1}
\end{array}\right]=\left[\begin{array}{cc}
T_{11} \rho_{0} c_{0} & T_{12}{ }^{\rho_{0} c_{0}} \\
T_{21} \rho_{0} c_{0} & T_{22} \rho_{0} c_{0}
\end{array}\right]\left[\begin{array}{c}
P_{2} \\
\rho_{0} c_{0} U_{2}
\end{array}\right]
$$

Where the relationship between particle velocity $U$ and volume velocity $Q$ is

$$
Q=U S
$$

Dividing a complicated acoustic system into several subsystems for which the transfer matrix model can be easily developed facilitates the derivation of the transfer matrix representation ${ }^{[5]}$. The transfer matrix of the Helmholtz resonator is comprised of the following three components: area contraction, area expansion and duct element.

The matrix of the area contraction is given by

$$
\left[\begin{array}{c}
P_{5} \\
\rho_{0} c_{0} U_{5}
\end{array}\right]=\left[\begin{array}{cc}
1 & \zeta_{4} \\
0 & A_{4} / A_{5}
\end{array}\right]\left[\begin{array}{c}
P_{4} \\
\rho_{0} c_{0} U_{4}
\end{array}\right]
$$

where $A_{4}$ is the cross-sectional area of the neck of the Helmholtz resonator, $A_{5}$ is the cross-sectional area of the tube, $\zeta_{4}$ is the normalized specific acoustic impedance

$$
\zeta_{4}=\frac{1}{\rho_{0} c_{0}}\left(\sqrt{2 \mu \rho_{0} \omega}+\frac{\rho_{0} \omega^{2} A_{4}}{2 \pi c_{0}}\right)+\frac{j 8 k r}{3 \pi}
$$

where the $k$ is the wave number, $k=\omega / c_{0}, \mu$ is dynamic viscosity of the air.

The matrix of the area expansion is given by

$$
\left[\begin{array}{c}
P_{3} \\
\rho_{0} c_{0} U_{3}
\end{array}\right]=\left[\begin{array}{cc}
1 & \left(A_{2} / A_{3}\right) \zeta_{3} \\
0 & A_{2} / A_{3}
\end{array}\right]\left[\begin{array}{c}
P_{2} \\
\rho_{0} c_{0} U_{2}
\end{array}\right]
$$

where $\mathrm{A}_{3}$ is the cross-sectional area of the neck of the Helmholtz resonator, $\mathrm{A}_{2}$ is the cross-sectional area of the cavity, $\zeta_{3}$ is

$$
\zeta_{3}=\frac{1}{\rho_{0} c_{0}}\left(\sqrt{2 \mu \rho_{0} \omega}+\frac{\rho_{0} \omega^{2} A_{4}}{2 \pi c_{0}}\right)+\frac{j 8 k r}{3 \pi}\left(1-1.25 \frac{r}{R}\right)
$$

Assuming the wave field in the duct is isentropic, The matrix of the duct is given by

$$
\left[\begin{array}{c}
P_{2} \\
\rho_{0} c_{0} U_{2}
\end{array}\right]=\left[\begin{array}{cc}
\cos (k L) & j \sin (k L) \\
j \sin (k L) & \cos (k L)
\end{array}\right]\left[\begin{array}{c}
P_{1} \\
\rho_{0} c_{0} U_{1}
\end{array}\right]
$$

The transfer matrix of the Helmholtz resonator can be written as

$$
\left[\begin{array}{c}
P_{1} \\
\rho_{0} c_{0} U_{1}
\end{array}\right]=T_{1} T_{2} T_{3}\left[\begin{array}{c}
P_{5} \\
\rho_{0} c_{0} U_{5}
\end{array}\right]
$$


where $T_{1}, T_{2}$, and $T_{3}$ are respectively the transfer matrices of the three parts. $T_{1}$ is the transfer matrix of the straight duct, $T_{2}$ is the transfer matrix of the neck and $T_{3}$ is the transfer matrix of the cavity.

\section{The FEM model analysis}

The three-dimensional finite element method (FEM) was used to simulate the performance of HR muffler. The comparison between simulation and theoretical results were analyzed.

According to the theory of acoustic waves, each duct has a corresponding to the cut-off frequency. When the vibration frequency of the sound source is less than the cut-off frequency of duct, the duct can only transmit the uniform plane wave. At low frequencies, the dimensions of the HR are much smaller than the wavelength of interest. Assuming the wall of the cavity is rigid, and the incident and emergent side is plane wave radiation. Then choose the analysis of frequency domain.

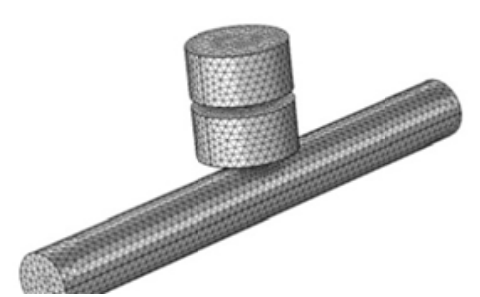

(a)series

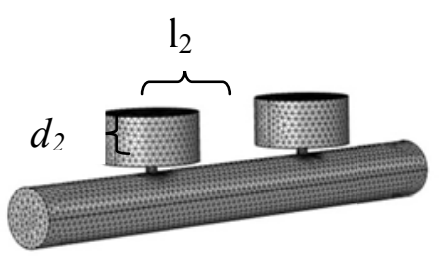

(b)parallel

Fig.2 FEM model of Helmholtz resonator acoustic Table 1 Physical dimensions of HR

\begin{tabular}{cccc}
\hline $\mathrm{d}_{1}$ & $\mathrm{~d}_{2}$ & $1_{1}$ & $1_{2}$ \\
\hline $10 \mathrm{~mm}$ & $90 \mathrm{~mm}$ & $10 \mathrm{~mm}$ & $45 \mathrm{~mm}$ \\
\hline
\end{tabular}

Fig.2(a) shows the 2 degrees of freedom Helmholtz resonator, which has two cylindrical necks and cavities connected in series. And Fig.2(b) shows the structure of 2 Helmholtz resonators connected in parallel. Where $d_{1}$ and $l_{1}$ are the lengths and diameter of necks, $d_{2}$ and $l_{2}$ are the lengths and diameter of cavities respective.

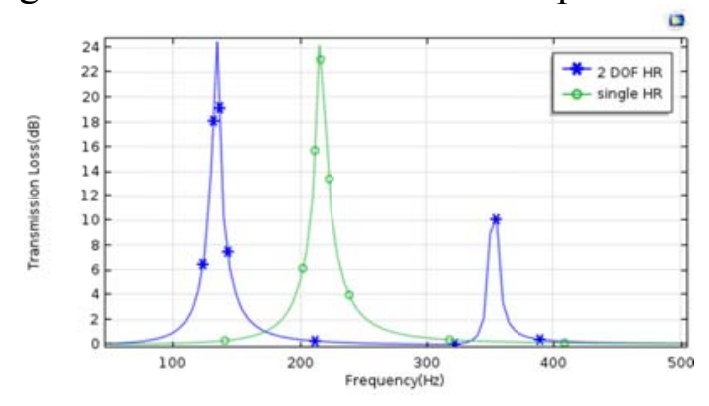

(a)

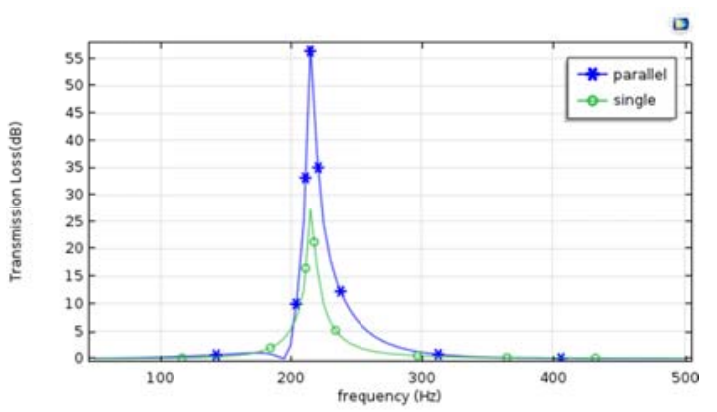

(b)

Fig.3. (a)The transmission loss of 2 DOF HR; (b)The transmission loss of 2 Helmholtz resonators connected in parallel

As shown in fig.3 (a), the 2 degrees of freedom Helmholtz resonator has two peak values of transmission loss, which beside the resonance frequency of the original single Helmholtz resonator. One resonance frequency moves to low frequency, another shifts to the high frequency.

As shown in fig.3(b), if choose the arrangement of 2 Helmholtz resonators connected in parallel, the transmission loss will be overlap on the original resonance frequency, which means that the performance of muffler is enhanced.

It is can be seen that when 2 Helmholtz resonators connected in parallel, the narrow-band not be changed and the performance of muffler is enhanced. As for the 2 degrees of freedom Helmholtz resonator, the narrow-band has been broaden. 


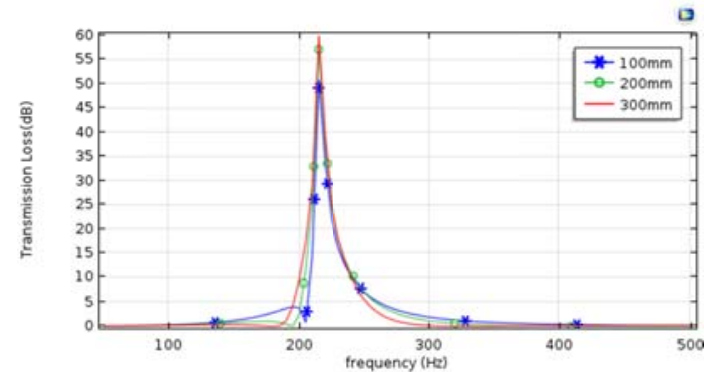

(a)

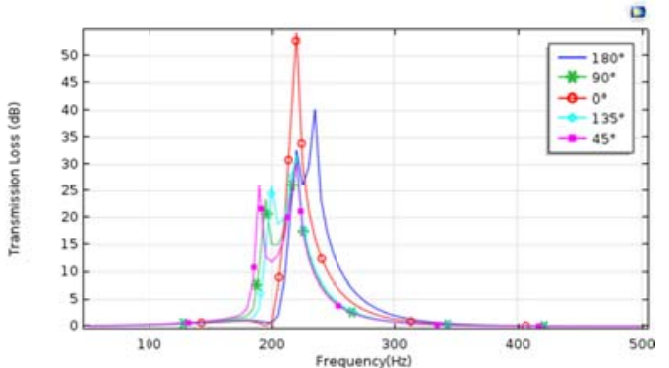

(b)

Fig.4. (a)The effect of distance between two Helmholtz resonators; (b) The effect of angle between two Helmholtz resonators

As shown in fig.4(a), we can neglect each transfer matrix and the distance between any two nearby resonators. The performance of muffler has no significant change with distance change.

As shown in fig.4(b), we can find that the effect of two Helmholtz resonators has been changed by the angle between the two Helmholtz resonators. The best effect of angle is 0 and 180, the angle of 90's absorption effect is the worst. Hence, it can be concluded that in the installation of a plurality of Helmholtz resonator, it is necessary to try to install on the same plane and avoid absorption effect caused by the angle decreased.

\section{Conclusions}

This paper use three-dimensional acoustic finite element method to calculate the acoustic characteristics of Helmholtz's resonant muffler. And the results show that the resonance frequency of the HR can be effectively enhanced by changing the arrangement of Helmholtz resonators.

The 2 degrees of freedom Helmholtz resonator has two resonance frequencies, and the narrowband has been broaden. The 2 Helmholtz resonators connected in parallel can significantly increase the noise elimination effect and the frequency of the noise is constant. It means that the narrowband not be changed and the performance of muffler is enhanced.

As for the effect of distance between two Helmholtz resonators, the performance of muffler no significant change with distance change.

Final, the best effect of angle is 0 and 180, the angle of 90's absorption effect is the worst.

\section{Acknowledgement}

This work is supported by the National Natural Science Foundation of China (NSFC, Grant No. 51305409) and Natural Science Foundation of Shanxi(Grant No. 2013021020-2)

\section{References}

[1] Liu Wenwen. Research on acoustical performance of Helmholtz muffler. Harbin Institute of Technology, 2010.

[2] Xiaofeng Shi, Cheuk Ming Mak. Helmholtz resonator with a spiral neck. Applied Acoustics, 2015, 99,68-71.

[3] Guan Changbin, Jiao Zongxia. Modeling and Optimal Design of 3 Degrees of Freedom Helmholtz Resonator in Hydraulic System. Chinese Journal of Aeronautics, 2012, 25, 776-783.

[4] Fei Liu. A tunable electromechanical Helmholtz resonator. University of Florida, 2007.

[5] Munjal ML. Acoustics of ducts and mufflers. John Wiley \& Sons, 2014. 